\title{
Commentary
}

\section{A role for IL-18 in intestinal inflammation?}

There is considerable evidence that inflammatory bowel disease (IBD), and a variety of other inflammatory disorders, reflect an excessive Th1 response. However, as we shall see below, a number of studies depleting the known members of this inflammatory cascade have reported some "residual" pathology and suggested that "other mediators" may also be involved. A study by Chikano and colleagues ${ }^{1}$ suggests that interleukin 18 (IL-18) may be one of the villains.

The characteristic cytokine produced by Th1 cells, interferon $\gamma(\mathrm{IFN}-\gamma)$, has been shown to play a role in the pathogenesis of a number of autoimmune and inflammatory disorders ${ }^{2}$ and has long been suspected of a role in IBD. Administration of IFN- $\gamma$ produces small intestinal pathology in mice ${ }^{34}$ while elevated IFN- $\gamma$ expression and increased numbers of IFN- $\gamma$ secreting cells have been reported in human IBD and are a feature of many of the murine models of IBD. ${ }^{56}$ The importance of IFN- $\gamma$ in such situations has been highlighted by the ability of in vivo depletion of this cytokine to ameliorate disease in a number of models of gastrointestinal pathology. However, more recent studies in a variety of models of IBD have revealed some inconsistencies. ${ }^{57}$ Some reports have suggested IFN- $\gamma$ knockout $(\mathrm{KO}) \mathrm{CD}^{+} \mathrm{T}$ cells are incapable of inducing colitis and wasting, others have reported comparable disease in IFN- $\gamma^{\text {null }}$ and wild type (WT) animals, while a recent study ${ }^{7}$ suggests that IFN- $\gamma$ KO T cells cause less severe pathology than that seen with WT cells. The study of Chikano and colleagues ${ }^{1}$ in last month's issue of Gut indicates that the pathological effects of combined IL-12 and IL-18 administration are similarly IFN- $\gamma$ dependent as they are not apparent in IFN- $\gamma \mathrm{KO}$ animals (see Gut 2000;47:779-86). However, the mechanism of the effects of interleukin 12 (IL-12) are not clear as they could not be mimicked by administration of exogenous IFN- $\gamma$ alone. It therefore seems likely that the IFN- $\gamma$ dependent effects of co-administration of IL-12 and IL-18 require the presence of additional mediators.

IL-12 production by activated macrophages and dendritic cells is one of the most important factors influencing polarisation of precursor T cells towards a Th1-type. ${ }^{8}$ This cytokine can cause enteropathy when administered to mice, ${ }^{4}$ is upregulated in human IBD, and its depletion prevents pathology in the murine TNBS colitis model. ${ }^{56}$ While the current study ${ }^{1}$ did not find intestinal pathology when IL-12 alone was administered, it is consistent with earlier findings using in vivo antibody depletion of IL-12 or animals deficient in the IL-12 signalling cascade molecule Stat-4, demonstrating that IL-12 is important, although not essential, for the development of colitis and that IL-12 dependent pathology may not require the presence of IFN $-\gamma .{ }^{9}$ The authors of this study proposed that the milder colitis that can develop in the absence of IL-12 may impli- cate other molecules, such as IL-18, which they had not investigated.

The recently discovered proinflammatory IL-1 family member IL-18 is produced by monocytes/macrophages, including liver Kupffer cells, and keratinocytes and has been shown to potentiate the effects of lipopolysaccharide and IL-12. ${ }^{10}$ IL-1 8 synergises with IL-12 in the induction of $\mathrm{T}$ cell priming, by sustaining the expression of the IL-12 receptor subunit IL-12R $\beta 2$, as well as production of the macrophage activating cytokine IFN $-\gamma$. This follows upregulation of IL-18R by IL-12, specifically on Th1 cells, suggesting that IL-18 is an important component of $\mathrm{T}$ cell priming leading to Th1 development. IL-18 increases IFN- $\gamma$ expression by stimulating activation of the transcription factors nuclear factor $\kappa \mathrm{B}$ and $\mathrm{AP}-1 .{ }^{10}$ Furthermore, IL-18 has been reported to be upregulated in IBD patients ${ }^{11}$ and a number of other inflammatory conditions. Thus the results of Chikano and colleagues ${ }^{1}$ are consistent with the suggested role of Th1 cells/IFN- $\gamma /$ IL-12 in enteropathy. ${ }^{56}$ However, this cytokine has also recently been shown to upregulate the Th2 polarising cytokine IL-13 and to potentiate Th2 responses in a number of models. ${ }^{12}{ }^{13}$ While Th1 and Th2 responses are usually considered to be mutually opposing aspects of $\mathrm{T}$ cell function, the recent description of apparently Th2 mediated IBD $^{5}$ indicates that Th2-type responses may contribute to intestinal pathology and it will be important to investigate the role of IL-18 in this context.

Tumour necrosis factor $\alpha(\mathrm{TNF}-\alpha)$ is one of the important effector molecules associated with the inflammatory cascade induced by activation of Th1 cells in a variety of autoimmune/inflammatory disorders and more recently in Th2 mediated pathology. ${ }^{5}$ TNF- $\alpha$ has also received much attention in IBD. ${ }^{5}{ }^{6}$ Increased TNF- $\alpha$ production has been reported in Crohn's patients and is also a feature of all of the murine models of IBD. ${ }^{45}$ Anti-TNF- $\alpha$ has been shown to reduce pathology and mortality in some of these systems ${ }^{56}$ while TNF- $\alpha$ causes significant enteropathy when administered to normal animals. $^{34}$ These studies have culminated in the successful use of anti-TNF- $\alpha$ therapy in Crohn's disease. $^{56}$ Thus TNF- $\alpha$ would have been a likely candidate as one of the "additional mediators" involved in the IL-12/18 induced, IFN- $\gamma$ dependent pathology described by Chikano and colleagues. It is therefore somewhat surprising that Chikano et al did not find a role for this molecule in the IL-12+IL-18 induced pathology they describe.

Another mediator usually associated with the Th1 mediated, $\mathrm{TNF}-\alpha$ associated inflammatory cascade is nitric oxide (NO). The role of NO in inflammatory conditions (particularly in the gastrointestinal tract) remains controversial and the debate is confounded by the multiple effects of this molecule on functions ranging from blood supply to direct damage via induction of apoptosis. However, NO appears to play a crucial role in a number of pathological conditions, including diabetes mellitus, graft versus host disease, rheumatoid arthritis, and several models of enteropathy. ${ }^{14}$ 
Thus Chikano et al show that IL-18 in combination with IL-12 can induce marked intestinal and hepatic pathology in an IFN- $\gamma$ dependent manner but that the majority of these effects are TNF- $\alpha$ and NO independent. Furthermore, while their studies demonstrating an absence of pathology when IFN- $\gamma$ is administered to mice contradict earlier work ${ }^{3}{ }^{4}$ they imply that the IFN- $\gamma$ dependent effects of IL-12/18 treatment are not mediated directly. These studies have important implications if considering IL-18 as a therapeutic target as this may be inappropriate in situations where TNF- $\alpha$ has a proven role, yet it may provide an adjunct or combination therapy where $\mathrm{TNF}-\alpha$ depletion alone has proved unsuccessful. However, before clinical applications are seriously considered, a number of issues will require clarification. It will be important to determine the mechanisms of the putative indirect effects via other receptor-ligand pairs which have yet to be investigated. In this respect the use of tissue/cell specific KOs of IFN- $\gamma / \mathrm{IFN}-\gamma \mathrm{R}$ will be invaluable in determining the sources and targets of IL-12, IL-18, and IFN- $\gamma$ and this will also aid identification of the "additional mediators" involved. Finally, the effects of depleting IL-18 (either with antibodies, soluble receptors, competitors, or via genetic manipulation) in models of IBD, or of IL-18 administration in Th2 models of IBD or in conjunction with Th2 cytokines will be essential.

Department of Immunology and Bacteriology, University of Glasgow, Western Infirmary, Glasgow G11 6NT, UK

paul.garside@clinmed.gla.ac.uk

P GARSIDE
The author is supported by the Wellcome Trust, MRC, BBSRC, ARC, SHERT, Robertson Trust, and Leverhulme Trust and is indebted to Professor Allan Mowat for critical review of the manuscript.

1 Chikano S, Sawada K, Shimoyama T, et al. IL-18 and IL-12 induce intestinal inflammation and fatty liver in mice in an IFN- $\gamma$ dependent manner. Gut 2000;47:779-86.

2 O'Garra A. Cytokines induce the development of functionally heterogeneous T helper cell subsets. Immunity 1998;8:275-83.

3 Garside P, Bunce C, Tomlinson RC, et al. Cytokine 1993;5:24-30.

4 Guy-Grand D, DiSanto JP, Henchoz P, et al. Small bowel enteropathy: role of intraepithelial lymphocytes and of cytokines (IL-12, IFN- $\gamma$, TNF) in the induction of epithelial cell death and renewal. Eur f Immunol 1998;28:730ind 44 .

5 Garside P. Cytokines in experimental colitis. Clin Exp Immunol 1999;118: 337-9.

6 MacDonald TT. Effector and regulatory lymphoid cells and cytokines in mucosal sites. Curr Top Microbiol Immunol 1999;236:113-35.

7 Bregenholt S, Brimnes J, Nissen $\mathrm{MH}$, et al. In vitro activated $\mathrm{CD}^{+}{ }^{+} \mathrm{T}$-cells from IFN- $\gamma$-deficient mice induce intestinal inflammation in immunodeficient hosts. Clin Exp Immunol 1999;118:228-34.

8 Abbas AK, Murphy KM, Sher A. Functional diversity of helper T lymphocytes. Nature 1996;383:787-93.

9 Simpson SJ, Shah S, Comiskey M, et al. T cell-mediated pathology in two models of experimental colitis depends predominantly on the interleukin 12/signal transducer and activator of transcription (Stat)-4 pathway, but is not conditional on interferon- $\gamma$ expression by T cells. $\mathcal{f} \operatorname{Exp}$ Med 1998;187: 1225-34.

10 Dinarello CA. Interleukin-18. Methods 1999;19:121-32.

11 Pizzaro TT, Michie MH, Bentz M, et al. IL-18, a novel immunoregulatory cytokine, is upregulated in Crohn's disease: expression and localization in intestinal mucosal cells. F Immunol 1999;162:6829-35.

12 Hoshino T, Wiltrout RH, Young HA. IL-18 is a potent coinducer of IL-13 in NK and T cells: a new potential role for IL-18 in modulating the immune response. F Immunol 1999;162:5070-7.

13 Wild JS, Sigounas A, Sur N, et al. IFN-gamma-inducing factor (IL-18) increases allergic sensitization, serum IgE, Th2 cytokines, and airway eosinophilia in a mouse model of allergic asthma. F Immunol 2000; 164:270110 .

14 Piedrafita D, Liew FY. Nitric oxide: A protective or pathogenic molecule? Rev Med Microbiol 1998;9:179-89.

\section{Autoimmune hepatitis: a fertile field}

The impact of chronic liver disease on the endocrine system has been of great interest to both endocrinologists and hepatologists, as multiple abnormalities in sex hormone metabolism have been reported. In addition, a number of clinical manifestation of liver disease have been reported to be the result of these, including amenorrhoea, impotency, gynaecomastia, and spider angiomata

There is probably no greater demand on the female endocrine system than the establishment and maintenance of pregnancy. In this regard, the disease autoimmune hepatitis is of particular interest as it predominantly impacts women, and at a relatively younger age, compared with many other forms of chronic liver disease. In addition, these patients often have cirrhosis by the time a diagnosis is made.

The majority of patients respond well to immunosuppressive drugs but most women with autoimmune hepatitis require maintenance immunosuppression for a sustained benefit. It has long been believed that women with autoimmune hepatitis have difficulty conceiving and maintaining pregnancy. Moreover, it has been reported previously that obstetric outcomes can be poor, with a relatively high incidence of toxaemia, premature delivery, and low birth weight.
In this issue of Gut, Heneghan and colleagues ${ }^{1}$ from the Institute of Liver Studies at King's College Hospital report the outcomes of 35 pregnancies in 18 women with autoimmune hepatitis (see page 97). This currently represents the largest reported series of pregnancies in women with autoimmune hepatitis. The report represents all known pregnancies in the autoimmune hepatitis population at King's College over a 15 year period.

The study is important in that it debunks the myth that pregnancy rarely occurs in women with autoimmune hepatitis or that there is a marked increase in the risk of fetal loss. From these 35 pregnancies, there were 31 live births. More than one third of patients had a diagnosis of cirrhosis that was made prior to conception. Two patients experienced, what has been previously reported, autoimmune hepatitis beginning during pregnancy. There were flares in disease activity in four pregnancies, and in another four patients within three months of delivery. The one disturbing fact was that there were two deaths, one of which occurred during pregnancy and one shortly after pregnancy.

The immunosuppressive agents used to control autoimmune hepatitis, azathioprine and corticosteroids, have been associated with concerns about potential birth defects. However, only two of 31 children developed birth defects that might be attributable to autoimmune hepatitis or its treatment. One child was delivered prematurely and had severe disabilities, which likely reflected the premature delivery. Another child had Perthe's disease. 
Women with autoimmune hepatitis of childbearing age and physicians who care for them must interpret this study as good news. I suspect there has been a longstanding admonition to these women that they should avoid pregnancy based on previously reported studies. Clearly, this study demonstrates that women with autoimmune hepatitis can become pregnant and can carry successful pregnancies to term with the expectation of delivering a normal baby.

There are however several caveats. Firstly, their disease needs to be monitored closely during this period because of the possibility of flare during pregnancy. Secondly, those with cirrhosis may experience complications of portal hypertension, such as variceal bleeding. Again, this needs to be monitored closely. Finally, it goes without saying that such patients need to be managed by physicians with real expertise in managing this disease. The group at King's College has extensive experience with this disease, and whether their excellent results will be obtained in the greater community at large remains to be determined. None the less, there are many liver centres throughout the world that are more than adequately equipped to manage these challenging patients. It is clear that these patients should be maintained during their pregnancy on immunosuppressive drugs at the lowest dose possible to maintain remission. Hence in addition to expert obstetrical management, our young female patients with autoimmune hepatitis can be expected to have families. This is truly important to young women with this disease who, with current therapies, can have longstanding periods of remission and a favourable natural history. Moreover, even for those women who fail therapy, liver transplantation is now an excellent alternative, such that these women should fully expect to not only raise their own families but see their children raise families as well.

J R LAKE

Division of Gastroenterology, Hepatology and Nutrition,

University of Minnesota, Minnesota, USA

1 Henegan MA, Norris SM, O'Grady JG, et al. Management and outcome of pregnancy in autoimmune hepatitis. Gut 2000;48:97-102.

\section{HCV in healthcare workers: a lightning strike}

In order to plan the control of hepatitis $C$ in our community, both in the general population and in hospitals, we need a clear picture of the prevalence and changing incidence of the infection in the overall population and in various subgroups.

The prevalence of hepatitis $\mathrm{C}$ virus (HCV) infection in the UK general population is not well established. Seroprevalence rates in established blood donors are very low $(0.002 \%)^{1}$ indicating a low incidence of infection at the present time, after institution of blood screening and an increased general awareness of $\mathrm{HCV}$ in the medical community. However, this is a highly selected population and gives no indication of the prevalence in the general population. New blood donors are also selected, being devoid of groups at high risk of acquiring blood transmitted viruses-in this group the seroprevalence rate is $0.06 \%{ }^{1}$

In a small study of the general population in south London, the prevalence of HCV viraemia was $0.7 \%{ }^{2}$ and in an unpublished study of the general population examined in a "DOH Look-Back" study in the southwest of England (subjects operated on by a HCV positive surgeon), a prevalence rate of $0.3 \%$ was observed. All of these values suggest that although the prevalence of $\mathrm{HCV}$ is lower in the UK than elsewhere in Europe and North America, it is still significant, suggesting that there are several hundred thousand people with this persistent infection.

The study of Thorburn and colleagues from Glasgow in this issue of Gut reports that the prevalence in healthcare workers is $0.28 \%,{ }^{3}$ similar to that observed in previous studies in healthcare workers ${ }^{45}$ and in the general population both in Glasgow $(0.24 \%)^{3}$ and in southwest England
$(0.28 \%)$, suggesting a low overall rate of transmission from patients to healthcare workers (see page 116). The subgroup analysis showed that the prevalence rate in those carrying out "exposure prone procedures" (invasive procedures where the hands or fingers of the surgeon, nurse, or other staff are in a patient's wound, and where there is a risk of injury to the worker that may result in exposure of the patient's open tissues to the blood of the worker), principally surgeons, was $0.23 \%$, similar to those not involved in these procedures $(0.30 \%)$ and to the prevalence in the general population $(0.28 \%)$. In considering only those healthcare workers undertaking exposure prone procedures, the lifetime risk of a UK surgeon acquiring HCV infection was $1.4 \%$ ( 1 in 70 ) over a surgical career of 35 years (0.04 per 100 person years), similar to that calculated in France $(0.3-3.1 \%){ }^{6}$

What impact do these values have on the assessment of the risk of transmission from healthcare workers to their patients during exposure prone procedures? ${ }^{7}$ Using the Glasgow values, the risk of transmission from healthcare workers and infection of a patient during exposure prone procedures can be calculated as 1 in 150000 (the prevalence of $\mathrm{HCV}$ infection in healthcare workers ( 1 in 500$) \times$ transmission rate (1 in 300 in the recent DoH "Look-Back" studies, personal communication from Dr Martin Wale)) and the risk of developing chronic infection 1 in 187500 , assuming an $80 \%$ rate of viral persistence. The consequences of persistent infection are delayed in that increased liver specific mortality is not seen for 20 or more years and even then it is small (approximately 1 in 50 per year). The risk of dying from $\mathrm{HCV}$ infection acquired from a surgical procedure is probably less than 1 in a million, comparable with the risk of being killed by lightning. ${ }^{8}$

Epidemiology will always be an important approach to the control of disease and $\mathrm{HCV}$ is no exception. We know that hospital acquired infection is relatively infrequent and in the general population intravenous drug abuse is an important method of transmission. For the future we need 
to know much more about how infection spreads in the general community outside of intravenous drug use; in $40 \%$ of $\mathrm{HCV}$ infected people donating blood in the UK between 1993 and1995, the method of acquisition of the virus was unknown?!

Liver Centre, Imperial College School of Medicine,

H C THOMAS

St Mary's Hospital, London W2 1PG, UK

h.thomas@ic.ac.uk

1 Communicable Disease Report Surveillance of viral infections in donated blood: England and Wales 1999. CDR Weekly 1999;10 (No 33);296.

2 Sallie R, King R, Silva E, et al. Community prevalence of hepatitis C viraemia, a polymerase chain reaction study. F Med Virol 1994;43:111-14.
3 Thorburn D, Dundas D, McCruden EAB, et al. A study of hepatitis C prevalence in healthcare workers in the West of Scotland. Gut 2000;48: prevalen

4 Zuckerman J, Clewly G, Griffiths P, et al. Prevalence of hepatitis C antibodies in clinical health care setting. Lancet 1994;343:1618-20.

5 Neal KR, Dornan J, Irving WL. Prevalence of hepatitis C antibodies among health care workers of two teaching hospitals. Who is at risk? $B M \mathcal{F}$ 1997;314:179-80.

6 Yazdanpanah Y, Boelle P-Y, Carrat F, et al. Risk of hepatitis C virus transmission to surgeons and nurses from infected patients: model-based estimates in France. F Hepatol 1999;30:765-9.

7 Esteban JI, gomez J, Martell M, et al. Transmission of hepatitis C by a cardiac surgeon. New Engl f Med 1996;334:555-9.

8 BMA Guide to Living with Risk. London: Penguin Books, 1990.

9 Soldan K, Barbara JAJ, Heptonstall J. Incidence of seroconversion to positivity for hepatitis C antibody in repeat blood donors in England, 1993-1995. BMF 1998;316:1413-17.

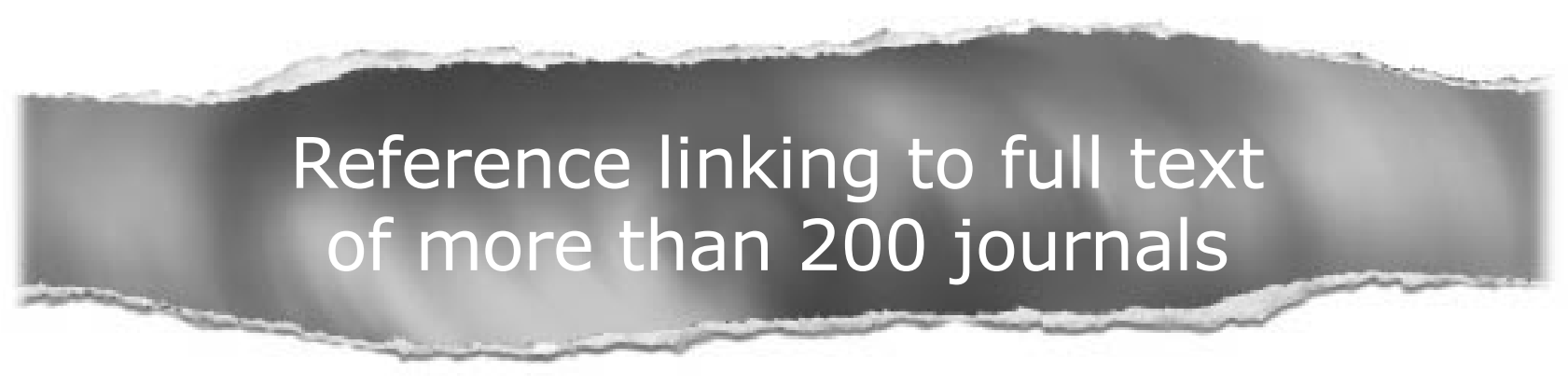

Toll free links

You can access the FULL TEXT of articles cited in Gut online if the citation is to one of the more than 200 journals hosted by HighWire (http://highwire.stanford.edu) without a subscription to that journal. There are also direct links from references to the Medline abstract for other titles.

www.gutjnl.com 